\title{
Untersuchungen über die elektrische Leitfähigkeit des Auges.
}

\author{
Von \\ Prof. E. Hertel, \\ I. Assistenten der Universitäts-Augenklinik zu Jena. \\ Mit einer Figur in Text.
}

\section{Einleitung.}

Die Bestimmung der elektrischen Leitfähigkeit des Körpers und seiner einzelnen Teile hat uns wertvollen Aufschluss über die Verwendbarkeit und Dosierung des elektrischen Stromes zu diagnostischen und therapeutischen $Z_{\text {wecken }}$ gebracht [v. Ziemssen (1), Erb (2), Stintzing(3)]; auch in der Elektropathologie ist die Kenntnis der Leitfähigkeit für den elektrischen Strom bei der Beurteilung und Verhütung von schädlichen Einwirkungen desselben von grosser Bedeutung [Jellineck (4)].

Besonderes Interesse hat aber in der neueren Zeit die Bestimmung der Leitfähigkeit gewonnen durch die namentlich von A rrhenius gegebene Aufklärung ihrer Beziehungen zu der chemischen Beschaffenheit des durchströmten Körpers [vgl. Hamburger(5)].

Der Gedanke von Faraday (1833), dass in Lösungen, welche den Strom leiten (Elektrolyten), die Moleküle gespalten sind in Teilmolekeln, die wandernd Träger der Elektrizität seien (daher Ionen), hatte in Hittorfs Analyse der an den Elektroden niedergeschlagenen chemischen Produkte eine wesentliche Stuitze gefunden. Kohlrausch hatte sodann auf Grund genaner Messungen der Grösse der Leitfähigkeit gefunden, dass diese bei hohen Verdünnungen der Lüsung bis zu einem gewissen, nicht überschreitbaren Maximum zunehme und dass sie gleich sei der Summe der Leitfähigkeiten der Ionen der Lösung, von deren Wanderungsgeschwindigkeit sie im wesentlichen abhängen sollte. Doch ergab sich bald und wurde auch von 
Kohlrausch gefunden, dass diese Erklärung der Vorgänge nicht für alle Fälle anwendbar sei.

Es war Arrhenius vorbehalten, Klarheit in diese Frage zu bringen. Nach ihm sind in den Elektrolyten stets in positive und negative Ionen gespaltene (dissoziierte) Moleküle vorhanden - ein Gedanke, den allerdings Clausius schon 1857 in ähnlicher Form ausgesprochen hatte -, und zwar entspricht dem jeweiligen Verdünnungsgrad der Lösung eine bestimmte Menge von dissoziierten Molekülen. Für den Transport der Elektrizität kommt nur dieser dissoziierte Teil in Betracht, es wird also um so mehr Elektrizität transportiert, d. h. die Leitfähigkeit um so besser sein, je grösser die Zahl der dissoziierten Moleküle ist, und diese ist um so grösser, je grösser die Verdünnung ist.

Es hängt demnach die Leitfähigkeit vor allem ab von dem Dissociationsgrad der Elektrolyten. Die Wanderungsgeschwindigkeit der Tonen kommt wesentlich nur dann in Betracht, wenn die Dissociation eine vollkommene ist, $d . h$. alle Moleküle in Ionen gespalten sind.

Somit haben wir also in der Bestimmung der Leitfähigkeit einen guten Anhaltspunkt, um uns über den Gehalt an dissoziierten Molekülen einer Flüssigkeit zu äussern, und damit einen nicht unwesentlichen Einblick in ihre Zusammensetzung und chemische Affinität zu ihrer Umgebung zu tun.

Für Blut, Lymphe, Harn, Galle usw. haben derartige Messungen wertvollen Aufschluss gebracht [Hamburger(5)]. Für den Humor aqueus des Auges sind Leitfähigkeitsbestimmungen noch nicht ausgeführt worden.

Aber auch die Leitfähigkeit des Auges in toto hat meines Wissens nach nur bei v. Ziemssen einige Berücksichtigung gefunden. Er erwähnt, dass sie sehr gross sei, ungefähr $\left.2^{2}\right|_{2}$ mal grösser als die der Muskeln, aber kleiner als die des Gehirns. Dieses oft citierte Resultat gewann v. Ziemssen auf Grund einer einzigen Bestimmung des absoluten Widerstandes des Auges und der zu vergleichenden Gewebe bei einem Kalbe. Leider sind die Bedingungen der Messung so ungenau angegeben, dass nicht zu ersehen ist, inwieweit wichtige, für die Messwerte ausschlaggebende Faktoren, auf die ich eingehend zurückkommen werde, berücksichtigt sind. Dadurch haben die ohnehin sehr spärlichen absoluten Zahlen von v. Ziemssen ihre Bedeutung so gut wie gänzlich verloren.

Meine eigenen Messungen erstreckten sich zunächst auf die spezifische Leitfähigkeit des Kammerwassers beim Kaninchenauge unter 
verschiedenen Bedingungen. Dann untersuchte ich aber auch die Leitfähigkeit des Auges in toto und zwar bei Kaninchen und Menschen.

\section{Versuchsanordnung.}

Bekanntlich ist die spezifische Leitfähigkeit eines Körpers für den elektrischen Strom umgekehrt proportional dem spezifischen Widerstand desselben. Den letzteren bestimmt man am einfachsten nach dem Prinzip der Wheatstoneschen Bricke, und zwar verwendete ich nach Kohlrausch, um die beim konstanten Strom wegen der Polarisation zu fürchtende Widerstandsänderung auszuschalten, Wechselströme zur Messung, die von einem kleinen Induktor mit sehr schnellen

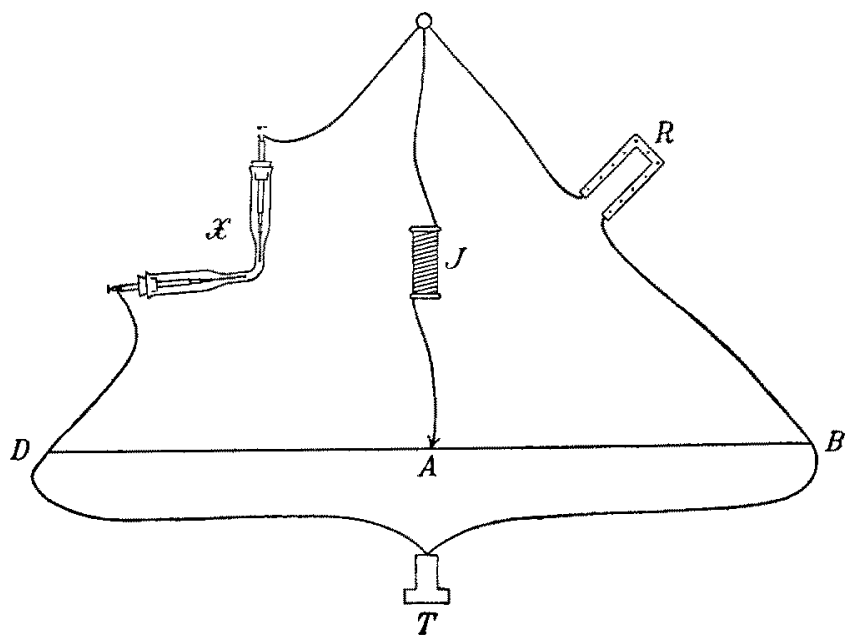

Unterbrechungen erzeugt wurden. Bei den Wechselströmen hebt sich die rasch und im entgegengesetzten Sinne erfolgende Polarisationswirkung auf und hat so auf den zu messenden Widerstand keinen Einfluss. Als Indikator für die Stromstärke wurde ein Siemenstelephon benutzt. Die beifolgende Skizze (vgl. Fig.) dürfte die Schaltung im übrigen leicht ersichtlich machen. Ist $R$ ein Rheostat von regulierbarer und bekannter Ohmzahl, $X$ der gesuchte Widerstand z. B. des Kammerwassers, dann lässt sich durch Verschieben von $A$ auf dem graduierten Brückendraht $B D$ nach $W$ hea ts tone stets eine Stellung finden, bei welcher durch das Telephon $T$ kein oder nur ein minimaler Strom geht, kenntlich durch das Verschwinden des Tones oder Auftreten eines Tonminimums. Haben wir diesen Punkt gefunden, dann verhält sich $X: D A=R: B A$, also

$$
X=R \cdot \frac{D A}{B A}
$$


somit ist $X$ bestimmt, sobald man die Stelle des Tonminimums auf der Skala $D B$ abgelesen hat. Die gesuchte Leitfähigkeit aber ist gleich dem reziproken Wert des Widerstandes, also $\lambda=1: X$.

Bekanntermassen ist nun die Leitfähigkeit eine Funktion der Temperatur der Körper, es musste also dieser grosse Aufmerksamkeit geschenkt werden. Ich habe daher die Gefässe mit dem zu untersuchenden Kammerwasser tief in ein Wasserbad gesenkt, dessen Temperatur an Thermometern mit 1/100 Gradteilung, deren Korrektionsfaktor in der physikalisch-technischen Reichsanstalt festgestellt war, genau abgelesen werden konnte. Ich bemerke dazu noch, dass die Gefässe mit dem Kammerwasser zunächst eine Viertelstunde lang in dem Wasserbad bei koustant eingestellter Temperatur gelassen wurden und dann stets mindestens zwei Messuagen in Pausen von je 10 Minuten angestellt wurden. Schwankungen der Temperatur bis zu ${ }^{5} / 100$ Grad glaubte ich als belanglos vernachlässigen zu können. In einigen Fällen (Kaninchen Nr. 4, 5 und 6) habe ich absichtlich die Temperatur verändert und grössere Messungsreihen angestellt, um den Einfluss der Temperatur genauer feststellen zu können.

Bei der Widerstandsmessung am lebenden Auge war die Temperatur von vornherein gegeben.

Die Grösse und Form des das Kammerwasser aufnehmenden Getässes ( $x$ in der Figur) war im iibrigen bestimmt durch die geringe Menge des zur Verfügung stehenden Kammerwassers. Ich liess mir aus Jenaer Normalglas stumpfwinklig abgebogene Röhren anfertigen, die an ihrer Umbiegungsstelle eine starke Verjüngung (Durchmesser ungetähr $5 \mathrm{~mm}$ ) hatten mit einer Graduierung in $\left.{ }^{1}\right|_{20} \mathrm{ccm}$. Ich hatte so stets die Kontrolle über die Menge der zu untersuchenden Flüssigkeit und zugleich auch genaue Anhaltspunkte für den naturgemäss stets gleich zu nehmenden Abstand der Elektroden. Diese wurden ein- für allemal so eingestellt, dass $2 / 10 \mathrm{ccm}$ Flüssigkeit $z$ wischen den selbst noch in die Flüssigkeit eingetauchten Elektrodenplatten stand.

Die Elektroden bestanden ganz aus Platin und hatten für die Kammerwassermessung einen Durchmesser von $4,5 \mathrm{~mm}$, sie liessen in der Röhre nur soviel Spielraum, dass sie bequem eingeführt werden konnten. Für die Bestimmung der Leitfähigkeit des ganzen Auges am lebenden Tier und Menschen benutzte ich ebenfalls Platinelektroden, die leicht gebogen, sich der Bulbusform gut anpassten in einer Grösse von 6:8 mm entsprechend der Grösse der Eulenburgschen Augenelektroden (6). Um das Tonminimum - möglichst exakt zu be- 
kommen, wurden die Elektroden gut platiniert, und zwar nach der Vorschrift von Lummer-Kurlbaum (7).

Die zur Feststellung des spezifischen Leitrermögens des Kammerwassers nötige Kenntnis der Widerstandskapazität des Flïssigkeitsbehälters endlich ermittelte ich für die betreffenden Temperaturen unter Benutzung einer maximalleitenden Bittersalzlösung von einem spezifischen Gewicht $=1,190$ gemessen bei 18 Grad. Es wurde davon $0,25 \mathrm{ccm}$ in das Röhrchen gegeben, die Elektroden genau so eingestellt, wie bei den Messungen mit Kammerwasser, und dann der Widerstand bestimmt. Multipliziert man diesen Wert mit dem spezifischen Leitvermögen der benutzten Flüssigkeit, ausgedrückt in reziproken $\mathrm{Ohm}$, so erhält man die Widerstandskapazität des Gefässes. Die Messungen gestalteten sich im einzelnen wie folgt.

I.

Temperatur im Rheostatén Tonminimum bei gesuchter Widerstand

(W 18)

\begin{tabular}{|c|c|c|c|}
\hline 18,02 & $80 \mathrm{ohm}$ & 509 & 82,88 \\
\hline 17,99 & 80, & 505 & 81,61 \\
\hline 18,01 & 80 & 505 & 81,61 \\
\hline 18,00 & 80 & 506 & 81,92 \\
\hline
\end{tabular}

$W_{18}$ in Mittel $=81,71$. Spezifisches Leitvermögen von $M g S O_{4}$ bei $18^{\circ}=$

$*_{18}=0,0492$ [nach Kohlrausch,Holbornu Diesselhorst(8)]; daraus ergibt sich die Widerstandskapazität des benutzten Gefässes bei $18^{\circ}=$

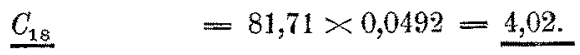

II.

$\begin{array}{cccc}\text { Temperatur } & \text { im Rheostaten } & \text { Tonminimum bei } & \text { gesuchter Widerstand } \\ 25,05 & 70 \mathrm{Ohm} & 500 & 70,00 \mathrm{Ohm} \\ 25,00 & 70 " \# & 501 & 70,29 \mathrm{~s} \\ 25,02 & 70 \% & 499 & 69,76, "\end{array}$

Mittelwert für $W_{25}=70,01 . \quad \varkappa_{25}=0,05808 ;$ also

$$
\underline{C_{25}}=70,01 \times 0,15808=\underline{4,066} \text {. }
$$

III.

$\begin{array}{cccc}\text { Temperatur } & \text { im Rheostaten } & \text { Tonminimum bei } & \text { gesuchter Widerstand } \\ 36,05 & 550 \mathrm{Ohm} & 505 & 56,32 \mathrm{Ohm} \\ 36,03 & 60 \% " & 489 & 57,41 \quad " \\ 36,00 & 60 \% & 490 & 57,64 \quad "\end{array}$

Mittelwert für $W_{38}=57,12 . \varkappa_{38}=0,07254$; also

$$
\underline{C_{36}}=57,12 \times 0,07254 \times \underline{4,14}
$$




\section{Messungsresultate der Kammerwasserleitfäbigkeit.}

Bevor ich die éinzelnen Messungsresultate anführe, möchte ich kurz noch auf die Materialgewinnung eingehen. Wir wissen, dass das Kammerwasser sehr leicht Veränderungen unterworfen ist, es genügen verhältnismässig schon geringfügige Eingriffe, um z. B. den Eiweissgehalt zu verändern [Wessely (9)]. Ferner wissen wir, dass Stoffe aus dem Conjunctivalsack, z. B. Kokain ziemlich schnell in das A ugeninnere diffundieren. Beide Momente konnten aber einen wesentlichen Einfluss auf die Leitfähigkeit des Kammerwassers haben und so die naturgemäss zunächst gesuchten Werte für das ganz normale Kammerwasser verändern.

Ich habe daher zuerst einige Vorversuche angestellt, um mich $z u$ überzeugen, inwieweit diese Befürchtung berechtigt war. Ich entnahm mit einer feinen Kanüle das Kanmerwasser bei demselben Tier aus dem einen Auge nach Kokainisierung (3 Tropfen einer $5 \%$ Lösung), und aus dem andern Auge unmittelbar nach Tötung des Tieres durch Schlag in den Nacken. Bei beiden Augen fand ich in zwei Versuchen den Eiweissgehalt gleich und innerhalb der normalen Grenzen, er war bei dem einen Tier nach Wessely bestimmt gleich $0,022 \%$ und bei dem andern gleich $0,03 \%$. Es war also ein Einfluss der beiden Eingriffe auf den Eiweissgehalt nicht erkennbar gewesen.

An weiteren Versuchen überzeugte ich mich dann, dass zur Entnahme des Kammerwassers die Instillation eines Tropfens Kokain in $5 \%$ Lösung genügt, und zwar konnte ich unmittelbar nach der Instillation schon die Kanüle einstechen, ohne dass das Tier, das ohne Fesselung auf dem Tisch sass, Abwehrbewegungen gemacht hätte oder das Auge sich injiziert hätte.

Ich bestimmte dann die Leitfähigkeit des Kammerwassers bei einem Tier, dessen eines Auge ich vor der Entnahme in oben angegebener Weise kokainisiert hatte und aus dessen anderem Auge ich das Kammerwasser unmittelbar nach der Tötung entnommen hatte. Die Zahlen gestalteten sich wie folgt:

Kaninchen Nr. I.

1. Kammerwasser aus dem kokainisierten Auge.

$\begin{array}{cccc}\text { Temperatur } & \text { im Rheostaten } & \text { Tonminimum bei } & \text { Kammerwasserwiderstand } \\ 25,02 & 270 & 490 & 259,41 \text { Ohm } \\ 25,01 & 260 & 503 & 262,08 \% " \\ 25,02 & 260 & 505 & 265,25 \% " \\ . & & & 9^{*}\end{array}$


Mittelwert des Kammerwasserwiderstandes bei $25^{\circ}=262,34$ Ohm

Widerstandskapazität des Gefässes bei $25^{\circ}=C_{25}=4,06$, also spezifische Leitfahigkeit des Kammerwassers bei $25^{\circ}=$

$$
\begin{aligned}
\lambda_{25}=\frac{4,066}{262,34} & =0,015501 \\
& =155,01 \cdot 10-4
\end{aligned}
$$

2. Kammerwasser des andern Auges sofort nach der Tötung entnommen.

$\begin{array}{cccc}\text { Temperatur } & \text { im Rheostaten } & \text { Tonminimum bei } & \text { Kammerwasserwiderstand } \\ 25,01 & 260 & 505 & 265,25 \mathrm{Ohm} \\ 25,00 & 260 & 500 & 260,00 \% " \\ 25,00 & 260 & 504 & 264,18 \% "\end{array}$

Kammerwasser-Leitfähigkeit bei $25^{\circ}=\lambda_{25}=\frac{4,066}{263,1}=0,015454$

$$
=154,54 \cdot 10^{-4}{ }^{1} \text {. }
$$

Daraus ergab sich, dass das Kokainisieren in angeführter Form keinen Einfluss auf die Leitfähigkeit des Kammerwassers gehabt hatte, ich konnte daher diese Methode unbedenklich bei weiteren Versuchen anwenden.

Die Tiere waren alle gesund und kräftig und hatten, wenn nicht ausdrücklich anderes bemerkt wird, vollkommen intakte Augen.

Kaninchen Nr. II.

I

Temperatur im Rheostaten Tonminimum bei Kammerwasserwiderstand

$\begin{array}{llll}36,01 & 220 & 502 & 220,88 \mathrm{Ohm} \\ 36,00 & 220 & 500 & 220,00 \%\end{array}$

Widerstand im Mittel $W_{36}=220,4 . \quad C_{36}=4,14$.

$\underline{\lambda_{36}}=187,94=$ Kammerwasser-Leitfähigkeit bei $36^{\circ}$.

II.

Temperatur im Rheostaten Tonminimum bei Kammerwasserwiderstand

$\begin{array}{llll}25,03 & 270 & 495 & 264,65 \mathrm{Ohm} \\ 25,00 & 270 & 496 & 265,70 \quad "\end{array}$

Widerstand im Mittel $W_{95}=265,13 . \quad C_{85}=4,066$.

$\underline{\lambda_{25}=153,36 .}=$ Kammerwasser-Leitfähigkeit bei $25^{\circ}$.

III.

Temperatur im Rheostaten Tonminimum bei Kammerwasserwiderstand

$\begin{array}{llll}18,01 & 300 & 506 & 307,29 \mathrm{Ohm} \\ 18,00 & 300 & 506 & 307,29 \quad,\end{array}$

Widerstand im Mittel $W_{18}=307,29 . C_{18}=4,02$.

$\underline{\lambda_{18}}=130,82=$ Kammerwasser-Leitfähigkeit bei $18^{\circ}$.

1) Der besseren Übersicht halber gebe ich im folgenden alle Schlusswerte für die Leitfähigkeit $10000 \mathrm{mal}$ zu gross wieder; es sind also alle Werte mit 10-4 multipliziert zu donken. 
Kaninchen Nr. III.

I.

Temperatur im Rheostaten Tonminimum bei Kammerwasserwiderstand

\begin{tabular}{lccc}
18,00 & 300 & 512 & $314,76 \mathrm{Ohm}$ \\
18,01 & 300 & 511 & $313,50 " ”$ \\
18,04 & 300 & 510 & $312,24 "$ \\
& $W_{18}=314,50 . \quad O_{18}=4,02$. & \\
& \multicolumn{2}{l}{$\lambda_{18}=127,82}$.
\end{tabular}

II.

Temperatur im Rheostaten Tonminimum bei Kammerwasserwiderstand

$\begin{array}{llll}25,00 & 300 & 479 & 275,82 \mathrm{Ohm} \\ 25,02 & 280 & 495 & 274,45 \mathrm{~N}\end{array}$

$W_{25}=275,18 . \quad C_{25}=4,066$.

$\underline{\lambda_{25}=147,77}$.

III.

Temperatur im Rheostaten Tonminimum bei Kammerwasserwiderstand

$\begin{array}{llcl}36,00 & 280 & 440 & 219,99 \mathrm{Ohm} \\ 36,00 & 280 & 439 & 219,10 ", \\ 36,01 & 280 & 440 & 219,99 " \\ & W_{18}=219,65 . C_{36}=4,14 . & \\ & & & \\ & & \end{array}$

Kaninchen Nx. IV.

I.

Temperatur im Rheostaten Tonminimum bei Kammerwasserwiderstand 18,00

$300 \quad 503$

$300 \quad 501$

$W_{36}=302,41 . \quad C_{18}=4,02$.

303,63 Ohm

18,00

$\underline{\lambda_{18}=132,93}$

II.

Temperatur im Rheostaten Tonminimum bei Kammerwasserwiderstand 25,00

25,02

25,20

265

265

265

25,30

270

501

500

499

495

301,20 "

26,30
$\stackrel{250}{W_{25}}=265,53 . \quad \stackrel{514}{C_{25}}=4,066$.
$\underline{\lambda_{25}}=153,13$.

266,06 $\mathrm{Ohm}$ \} Mittel

$265,00, \quad\}=265,53$

264,09 "

264,65 ,

$264,40 "$

III.

Temperatur im Rheostaten Tonminimum bei Kammerwasserwiderstand

$\left.\begin{array}{llll}34,15 & 210 & 509 & 217,07 \mathrm{Ohm} \\ 35,65 & 210 & 505 & 214,26 " \\ 36,00 & 210 & 502 & 211,68 " \\ 36,02 & 210 & 500 & 210,00 "\end{array}\right\}=2108$


Temperatur im Rheostaten Tonminimum bei Kammerwasserwiderstand $\begin{array}{llll}36,46 & 200 & 510 & 208,160 \mathrm{obm} \\ 37,15 & 250 & 450 & 204,55 \quad,\end{array}$

$$
\begin{gathered}
W_{36}=210,8 . \quad C_{36}=4,14 . \\
\underline{\lambda_{36}=196,45 .}
\end{gathered}
$$

\section{Kaninchen Nr..}

I.

Temperatur im Rheostaten Tonminimum bei Kammerwasserwiderstand

$\begin{array}{llc}36,01 & 220 & 485 \\ 36,00 & 220 & 465 \\ 35,05 & 220 & 487 \\ 34,25 & 270 & 430 \\ & W_{36}=209,17 . C_{36}=4,14 . \\ & & \\ & & \end{array}$

$209,17 \mathrm{Ohm}$ Mittel

$209,17, \quad\}=209,17$

205,52 "

203,68 "

II.

Temperatur im Rheostaten Tonminimum bei Kammerwasserwiderstand

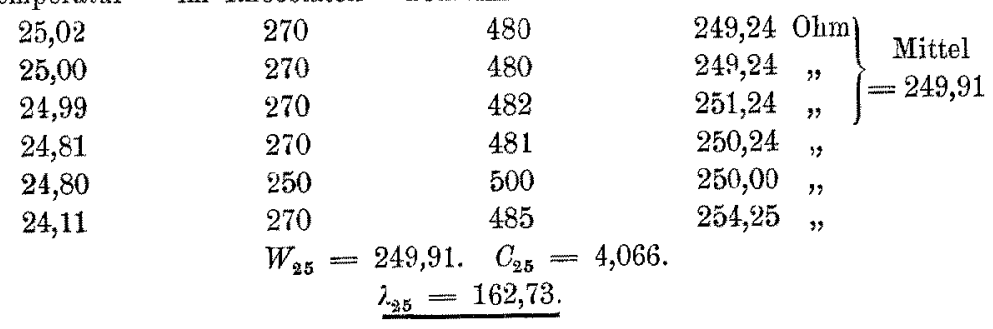

III.

Temperatur im Rheostaten Tonminimum bei Kammerwasserwiderstand

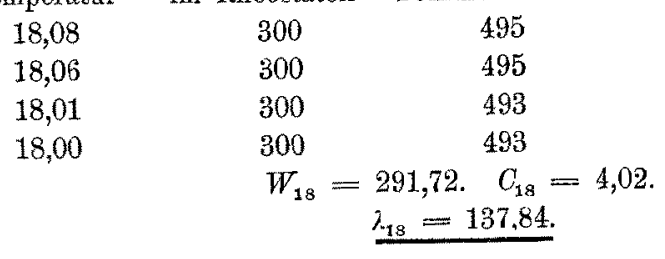

1. Kammerwasser aus normalem Auge. Eiweissgehalt: $0,029 \%$.

Temperatur im Rheostaten Tonminimum bei Kammerwasserwiderstand

18,02

18,00

18,00
Kaninchen Nr. VL.

$\begin{array}{ccc}300 & 506 & 306,29 \mathrm{Ohm} \\ 300 & 505 & 306,06 " \\ 300 & 505 & 306,06 " \\ W_{18}=306,1 . & C_{18}=4,02 . & \end{array}$

300

$W_{18}=306,1 . \quad C_{18}=4,02$. 294,06 Obm

294,06 "

$291,72, \quad$ Mittel

$291,72,\}=291,72$

$\underline{\lambda_{18}=131,33 .}$ 
2. Kammerwasser desselben Auges nach Punktion. Eiweissgehalt: $1,75 \%$. Temperatur im Rheostaten Tonminimum bei Kammerwasserwiderstand

\begin{tabular}{|c|c|c|c|}
\hline 18,02 & 270 & 550 & 329,99 \\
\hline 18,01 & 300 & 525 & 381,59 \\
\hline 18,00 & 300 & 524 & 330,24 \\
\hline
\end{tabular}

Kaninchen Nr. VII.

1. Kammerwasser aus dem normalen Auge. Eiweissgehalt: $0,03 \%$.

Temperatur im Rheostaten Tonminimum bei Kammerwasserwiderstand

$$
\begin{array}{lccc}
36,00 & 210 & 500 & 210,00 \mathrm{Ohm} \\
36,00 & 210 & 502 & 211,68 \% " \\
& W_{\mathbf{s e}}=210,80 . C_{36}=4,14 . & \\
& & \lambda_{3 e}=196,45 .
\end{array}
$$

2. Kammerwasser aus dem entzündeten Auge. Eiweissgehalt: 3,4\%.

Temperatur im Rheostaten Tonminimum bei Kammerwasserwiderstand

$$
\begin{array}{cccc}
36,00 & 250 & 500 & 250,00 \mathrm{Ohm} \\
36,01 & 250 & 500 & 250,00 \% " \\
36,01 & 250 & 499 & 249,15 \quad " \\
& W_{36}=249,72 . C_{36}=4,14 . & \\
& &
\end{array}
$$

Kaninchen Nr. VIII.

1. Kammerwasser ans dem normalen Auge. Eiweissgehalt: $0,021 \%$.

Temperatur im Rheostaten Tonminimum bei Kammerwasserwiderstand

$$
\begin{array}{llcl}
25,05 & 260 & 504 & 264,18 \mathrm{Ohm} \\
25,01 & 270 & 495 & 264,65 \quad " \\
& W_{25}=264,42 . C_{25}=4,066 . & \\
& \multicolumn{2}{c}{\lambda_{25}=153,78 .}
\end{array}
$$

2. Kammerwasser aus dem entzündeten Auge. Eiweissgehalt: 2,9\%.

Temperatur im Rheostaten Tonminimum bei Kammerwasserwiderstand

$$
\begin{array}{llcc}
25,00 & 300 & 505 & 306,06 \mathrm{Ohm} \\
25,63 & 300 & 506 & 309,63 \% " \\
& W_{35}=304,84 . C_{25}=4,066 . & \\
& & \\
& &
\end{array}
$$

Nach den angeführten Zahlen lagen also die Werte für die Kammerwasserleitfähigkeit:

$$
\begin{aligned}
& \text { bei } 18^{\circ} \mathrm{C} \text {. (5 Tiere) zwischen } 127,82 \text { und } 137,84 \text {, } \\
& " 25^{\circ} "(6 \quad ") \quad \text { " } 6153,13 \quad, 162,73 \text {, } \\
& \text { " } 36^{\circ} "(5 \quad ") \quad \text { " } \quad 187,94 \quad " 198,20 \text {. }
\end{aligned}
$$


Will man daraus einen Mittelwert berechnen, so wïrde sich derselbe:

$$
\begin{aligned}
& \text { für } 18^{\circ} \text { auf } 132,15, \\
& " 25^{\circ} \text { " } 154,37, \\
& " 36^{\circ}, 193,51 \text { stellen. }
\end{aligned}
$$

Man sieht deutlich auch beim Kammerwasser den Einfluss der Temperatur auf die Grösse der Leitfähigkeit, und zwar berechnete

\begin{tabular}{|c|c|c|c|c|c|}
\hline $\begin{array}{r}\text { Leitfä } \\
z u J\end{array}$ & $\begin{array}{l}\text { higkeits- } \\
\text { lahme }\end{array}$ & $\begin{array}{c}\text { Temp. -Zunahme } 7^{\circ} \\
\text { von } 18^{\circ}-25^{\circ}\end{array}$ & $\begin{array}{c}\text { Temp.-Zunahme } 11^{\circ} \\
\text { von } 25^{\circ}-36^{\circ}\end{array}$ & & \\
\hline bei & Tier 2 & $2,2 \%$ & $2,1 \%$ & im Mittel & $=2,15$ \\
\hline$"$ &,$\quad 3$ & $2,2 \%$ & $2,5 \%$ &,$\quad "$ & $=2,35$ \\
\hline$"$ & $"$ & $2,2 \%$ & $2,5 \%$ & $"$ & $=2,35$ \\
\hline$"$ & 5 & $2,1 \%$ & $2,5 \%$ & " & $=2,3$ \\
\hline
\end{tabular}
sich die prozentuale Zunahme bei den einzelnen Tieren in folgender Weise:

Daraus würde sich als Mittelwert 2,35\% ergeben.

Bei den Tieren 6 und 7 habe ich dann zur Kontrolle auch noch die Widerstandsänderung innerhalb kleinerer Temperaturschwankungen direkt gemessen und fand folgende Werte:

Bei Tier 4 u. einer Temp.-Änderg. v. $25,00^{\circ}-26,3^{\circ}$ eine Widerstandsabn. $=2,6^{\circ} \%$

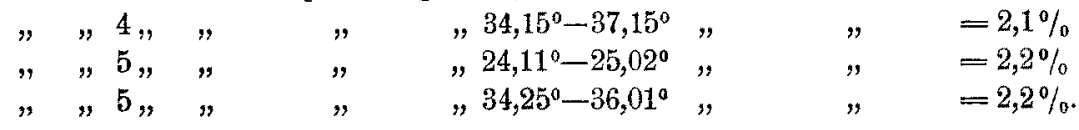

Es würde sich daraus das Mittel $=2,3 \%$ ergeben, ein mit dem vorher berechneten gut übereinstimmender Wert, so dass der Temperaturkoëffizient der Kammerwasserleitfähigkeit $=0,023$ gesetzt werden kann. Dieser würde sich also nicht wesentlich von dem des Serums unterscheiden, den Bugarszky und Tangl $(10)=0,022$ angegeben haben.

Um weitere Beziehungen zwischen der Leitfähigkeit des Kammerwassers und der des Serums zu finden, habe ich bei den Tieren 2, 3, 4 und 5 die Leitfähigkeit des Serums nach derselben Methode, wie ich sie für die Messungen beim Kammerwasser angewendet und genauer geschildert habe, bestimmt und zwar für eine Temperatur von $18^{\circ} \mathrm{C}$. Die gefundenen Werte sind in der ersten Kolumne der folgenden Tabelle enthalten; in der zweiten Kolumne stehen zum Vergleich die entsprechenden Werte der Leitfähigkeit des Kammerwassers für dieselben Tiere; auf die Zahlen in Kolumne 3 komme ich sogleich zu sprechen. 


$\begin{array}{cccc} & \begin{array}{c}\text { Serum- } \\ \text { leitfühigkeit } \\ \text { bei } 18^{0}\end{array} & \begin{array}{c}\text { Kammerwasser- } \\ \text { leitfähigkeit }\end{array} & \begin{array}{c}\text { Korrigierte Serum- } \\ \text { leitfuhigkeit }\end{array} \\ \text { Tier } 2 & \mathbf{1 1 0 , 2 9} & \text { bei } 18^{0} & \text { bei } 18^{\circ} \\ " 3 & 110,10 & 130,82 & 130,05 \\ " 4 & 114,82 & 127,82 & 130,01 \\ " 5 & 112,35 & 132,93 & 135,8 \\ & & 137,84 & 132,95 .\end{array}$

Ein Vergleich der beiden ersten Kolumnen ergibt ohne weiteres, dass die bei der Messung der Leitfähigkeit des Serums erhaltenen Zahlenwerte nicht unbeträchtlich hinter denen der Kammerwasserleitfähigkeit derselben Tiere zurückstehen.

Aus der chemischen Analyse des Kammerwassers und des Serums wissen wir nun, dass die Hauptdifferenz zwischen beiden in dem Eiweissgehalte liegt. Während derselbe beim Kaninchenkammerwasser nach Wessely (11) im Mittel $=0,025 \%$ ist, ein Steigen über $0,04 \%$ jedenfalls nicht mehr als normal zu betrachten ist, beträgt er beim Kaninchenserum nach Hammarsten (12) 6,25\%.

Es war nun sehr wohl möglich, dass diese Differenz im Eiweissgehalt auch die Differenz in der Leitfähigkeit der beiden Flüssigkeiten bedingt hatte. Denn wir wissen aus der Arbeit von Bugarszky und Tangl(10), dass ein jedes Prozent Eiweiss die Leitfähigkeit des Serums um 2,5\% herabsetzt. Unter Zugrundelegung dieses Wertes können wir die Höhe der durch den Eiweissgehalt in unsern Fällen bedingten Verminderungen der Leitfähigkeit berechnen nach folgender Formel:

$$
\lambda_{c}=\lambda \cdot \frac{100}{100-2,5.6,2},
$$

wobei $\lambda_{c}=$ der gesuchten Leitfähigkeit des Serums ohne Eiweiss und $\lambda=$ der gefundenen Leitfähigkeit des eiweisshaltigen Serums ist.

Die auf diese Weise erhaltenen Werte für die Leitfähigkeit des Serums nach Abzug des Einflusses des Eiweissgehaltes (korrigierte Leitfähigkeit) finden sich in der letzten Kolumne der vorstehenden Tabelle angeführt. Es ist unverkennbar, dass sie den für die Leitfähigkeit des Kammerwassers gefundenen Werten sehr ähneln; auf die kleinen Differenzen zwischen beiden möchte ich um so weniger Gewicht legen, als ja der Eiweissgehalt des Serums nur nach Mittelwerten in die Rechnung einbezogen worden ist.

Es würde sich also daraus der Schluss ergeben, dass die Kammerwasserleitfähigkeit gleich ist der korrigierten Serumleitfähigkeit desselben Tieres. 
Nur eines möchte ich noch erwähnen, dass eine gleiche Korrektur hinsichtlich des Eiweissgehaltes auch beim Kammerwasser vorzunehmen, mir überflüssig erschienen ist, da die in Betracht kommenden Werte viel zu gering sind. So würde z. B. der aus den Messungen sich ergebende Mittelwert für die Leitfähigkeit bei $18^{\circ}$ nur von 132,15 auf 132,24 steigen selbst unter Zugrundelegung von $0,04 \%$ Eiweiss im Kammerwasser. Es würde also die Vornahme der Korrektur keinerlei Einfluss auf das obige Gesamtresultat haben.

Durch die Punktion der Kammer und durch entzündliche Prozesse des Auges wird die Leitfähigkeit des Kammerwassers wesentlich vermindert, wie aus den Messungsresultaten bei den Tieren 6,7 und 8 hervorgeht. Und zwar konstatierte ich gegenüber den auf der normalen Seite festgestellten Werten eine Herabsetzung bei Tier 6 durch Punktion um 7,4\%, durch Entzündung bei Tier 7 um $15,5 \%$, bei Tier 8 um $15,3 \%$.

Im Hinblick auf die schon citierte Arbeit von Bugarszky und Tangl(10) lag es nahe, auch für diese Verminderung die Steigerung des Eiweissgehaltes verantwortlich zu machen, die wir ja als auffallendste Folge sowohl der Punktion der Kammer als auch der Entzündung des Auges kennen. Legen wir wieder die von Bugarszky und Tangl gemachte Beobachtung, dass eine Steigerung des Eiweissgehaltes um $1 \%$ die Leitfähigkeit um $2,5 \%$ vermindert, zugrunde, so müsste der aus der von uns gefundenen prozentualen Verminderung der Leitfähigkeit des Kammerwassers berechenbare Eiweissgehalt etwa gleich sein dem direkt, z. B. durch Fällung messbaren Eiweissgehalt.

Ich habe nun diese Rechnung durchgeführt, ferner den Kammerwassereiweissgehalt nach der'von Römer(13) angegebenen Methode (bezogen auf Serumlösung von bekanntem Eiweissgehalt) bestimmt und fand folgende Werte:

$\begin{array}{ccccc} & \begin{array}{c}\text { Verminderung der } \\ \text { Leitfähigkeit }\end{array} & \begin{array}{c}\text { Berechneter } \\ \text { Eiweissgehalt }\end{array} & \begin{array}{c}\text { Gemessener } \\ \text { Eiweissgehalt }\end{array} & \text { Differenz } \\ \text { Tier } 6 & 7,4 \% & 2,96 \% & 1,75 \% & 1,21 \\ " 7 & 15,5 \% & 6,2 \% & 3,4 \% & 2,8 \\ " 8 & 15,3 \% & 6,1 \% & 2,9 \% & 3,2\end{array}$

Es blieb also der direkt gemessene Eiweissgehalt nicht unbeträchtlich hinter dem berechnetẹn zurück. Begründet ist diese Differenz wohl vor allem durch die Tendenz des veränderten Kammerwassers zur Gerinnung. Die dadurch bedingte Konsistenzvermehrung kann die Ionenbewegung schon allein durch vermebrte Reibung behindern. $\mathrm{Ob}$ vielleicht ausserdem noch das ja als pathologisches Pro- 
dukt zu betrachtende Eiweiss auch auf die Dissociation der Moleküle und dadurch auf die Zahl der Ionen im Kammerwasser von Einfluss gewesen ist, müsste durch weitere Untersuchungen festgestellt werden.

\section{Messungsresultate der Bulbusleitfähigkeit.}

Bei Messungen der Leitfähigkeit am lebenden Körper ist vor allem zu berücksichtigen, dass der eingeführte elektrische Strom nicht nur das zwischen den Elektroden liegende zu messende Stïck oder Glied durchzieht, sondern in weiten Schleifen über die Nachbargebiete, meist wohl durch den ganzen Körper cirkuliert. Es wird natürlich diese nicht ohne weiteres zu ermittelnde Stromverzweigung das Resultat der Widerstandsmessung einzelner Teile des Körpers beeinflussen. Man könnte diese Stromschleifen nur vermeiden dadurch, dass man die zu messenden Organe aus ibrem Verband mit den Nachbarorganen herauslöst. $\mathrm{Zu}$ dieser Art der Messung, die z. B. auch bei der einzigen bisher vorliegenden Widerstandsbestimmung des Bulbus ron v. Ziemssen (1) angewendet worden ist, konnte ich mich nicht entschliessen. Denn die dadurch bedingte Aufhebung der Cirkulation in dern Organ, die Änderung der Temperatur, die mehr oder weniger schnell einsetzenden chemischen Veränderungen infolge der beginnenden Absterbeerscheinungen müssen sich jedenfalls auf die Leitfähigkeit von Einfluss erweisen, und zwar in nicht zu kontrollierender Weise. Lassen wir dagegen das Auge in situ und wählen die Elektrodenstellung in allen Versuchen gleich, so ist der Fehler durch die Stromschleifen, wenn auch nicht kontrollierbar, so doch als gleich gross zu setzen, da nicht anzunehmen ist, dass die Schleifen bei Tieren derselben Species wesentlich different verlaufen. Dazu kommt, dass wir auf diese Weise die Messungen anstellen können unter Bedingungen, die auch in der Praxis bei Verwendung der Elektrizität ain Auge in Betracht kommen können.

Für alle Versuche wurden die Elektroden nach Einlegung eines Lidsperrers eine Elektrodenbreite $=6 \mathrm{~mm}$ vom Limbus in der horizontalen Mittellinie angelegt. Die Elektroden hatten, wenu nichts anderes erwähnt ist, die Grösse der auch in die Praxis eingeführten Eulenburgschen Augenelektroden $(6: 8 \mathrm{~mm})$. Sie waren platiniert und wurden vor dem Anlegen in warmer $0,6 \% \mathrm{NaCl}$-Lösung angefeuchtet und feucht aufgelegt.

Der primäre, zum Induktor geleitete Strom war gleich 2 Volt.

Die Schaltung und Berechnung der gesuchten Werte wurde 
genau so ausgeführt wie bei der Leitfähigkeitsbestimmung des Kammerwassers, es dürfte sich daher das Eingehen auf die Details der Einzelversuche erubrigen.

Bei Kaninchen, es wurden bei sechs normalen Tieren Messungen angestellt, bewegten sich die Widerstandswerte zwischen 202 und $241 \mathrm{Ohm}$. Im Mittel berechnete sich daraus die Leitfähigkeit:

$$
\left.\lambda=\frac{1}{217,8}=45,4 \cdot 10^{-4} \mathrm{Ohm}^{-11}\right) \text {. }
$$

Bei vier von diesen Tieren konnte durch Vergleichsmessungen an beiden Augen festgestellt werden, dass die Kokainisierung des Auges (3 Tropfen einer 5\% Lösung) die Leitfähigkeit des Bulbus nicht verändert. Ebensowenig konnte bei den beiden letzten ein Einfluss einer Allgemeinnarkose (Äther, Chloroform) konstatiert werden.

Ich untersuchte sodann zwei Tiere mit Entzündung auf je einem Auge (Glaskörpereiterung nach Staphylocokkenimpfung). Ich fand bei dem ersten Tier auf der gesunden Seite:

auf der kranken:

$$
\lambda_{g}=\frac{1}{218}=45,6 \text {, }
$$

beim zweiten entsprechend:

$$
\lambda_{i}=\frac{1}{222}=45,0,
$$

$$
\lambda_{g}=\frac{1}{234}=42,5 \quad \text { und } \quad \lambda_{k}=\frac{1}{230}=43,5 .
$$

Es bestand also bei beiden Tieren keine eindeutige Differenz in der Leitfähigkeit zwischen dem gesunden und kranken Auge.

Bei diesen Tieren habe ich die Versuche noch weiter modifiziert. Ich bestimmte unter Benutzung von 4,5 mm messenden runden Platinelektroden nochmals den Widerstand der Bulbi, und eröffnete dann an den Anlegestellen der Elektroden den Glaskörper und wiederholte die Bestimmung nach Einführen der Elektroden in das Augeninnere, so dass sie soeben mit ihren Platten in den Glaskörper tauchten. Das gab folgende Werte:

$$
\text { gesunder Bulbus }
$$

Tier 1

" 2

$$
\begin{aligned}
& \lambda=\frac{1}{304}=32,9 \\
& \lambda=\frac{1}{317}=31,5
\end{aligned}
$$

kranker Bulbus

$$
\begin{aligned}
& \lambda=\frac{1}{305}=32,8 \\
& \lambda=\frac{1}{315}=31,8
\end{aligned}
$$

1) Es sollen auch bei diesen Messungen alle folgenden Leitfahigkeitswerte $10000 \mathrm{mal} \mathrm{zu}$ gross angegeben werden. 


$\begin{array}{cll} & \text { gesunder Glaskörper } & \text { kranker Glaskörper } \\ \text { Tier } 1 & \lambda=\frac{1}{196}=51,0 & \lambda=\frac{1}{256}=37,2 \\ , 2 & \lambda=\frac{1}{205}=48,8 & \lambda=\frac{1}{242}=41,3 .\end{array}$

Es waren also die Bulbuswiderstände bei beiden Tieren auf der kranken und gesunden Seite gleich und untereinander ziemlich ähnlich. Die höheren Zahlen gegenüber den vorhin skizzierten Werten erklären sich durch die kleineren Elektroden.

Dagegen fanden sich bei demselben Tier beträchtliche Unterschiede zwischen dem Widerstand im gesunden und im kranken Glaskörper. Bei Tier 1 betrug die Verminderung der Leitfähigkeit auf der kranken Seite $27 \%$, bei Tier $215,3 \%$. Ich komme auf diese Zahlen nochmals zurück.

Ich möchte vorher erst noch die Messresultate an Menschenaugen besprechen. Die Messungen wurden stets bei Erwachsenen ausgeführt mit Elektroden von $6: 8 \mathrm{~mm}$.

Nach Kokainisierung (3 Tropfen einer 5\% Lösung) habe ich zunächst an meinem linken Auge (normal, Myopie $=2,5 \mathrm{D}$ ) die Leitfähigkeit bestimmen lassen. Es wurde gefunden:

$$
\lambda=\frac{1}{302}=33,1 \text {. }
$$

Abgesehen von subjektiven Lichterscheinungen und leichtem Brennen, das nach Aufhören der Kokainwirkung sich bemerkbar machte, habe ich von der Messung nichts bemerkt.

Ich habe dann bei drei Männern im Alter von 20-30 Jahren mit normalen Augen dieselbe Messung ausgeführt und fand folgende Werte:

$$
\lambda_{1}=\frac{1}{301}=33,1 ; \quad \lambda_{2}=\frac{1}{326}=30,7 ; \quad \lambda_{3}=\frac{1}{310}=32,3 .
$$

Eine weitere Bestimmung stellte ich dann bei einem Manne in Narkose an. Die Leitfähigkeit seines gesunden Auges war:

$$
\lambda=\frac{1}{305}=32,7
$$

die des kranken, das wegen schwerer infektiöser Iridocyclitis mit Glaskörperveränderungen enucleiert werden sollte:

$$
\lambda=\frac{1}{306}=32,7 \text {. }
$$

Schliesslich wurde bei mir selbst noch eine Widerstandsbestim- 
mung bei geschlossenen Lidern gemacht. Dabei wurden die Elektroden fest aufgelegt und weit nach hinten gedrückt, um einen möglichst grossen Abstand zu erzielen. Der Widerstand war $=3660 \mathrm{Ohm}$, also die Leitfähigkeit $=2,72$.

Zwei gleiche Bestimmungen in genau derselben Weise fübrte ich an zwei Frauen aus und fand bei der einen:

$$
W=3294 \text { Ohm, also } \lambda=3,03,
$$

bei der andern:

$$
W=3375 \mathrm{Ohm}, \text { also } \lambda=2,96 .
$$

Ein Überblick über die mitgeteilten Messungsresultate lässt leicht erkennen, dass die bei den einzelnen Individuen derselben Spezies beobachteten Schwankungen in der Leitfähigkeit des Bulbus sich innerhalb relativ enger Grenzen bewegten. Der höchste bei Kaninchen gefundene Wert war $49,5 \cdot 10^{-4}$, der niederste $41,5 \cdot 10^{-4}$. Beim Menschen waren die entsprechenden Werte $33,1 \cdot 10^{-4}$ und $30,7 \cdot 10^{-4}$. Die aus allen Werten berechneten Mittel werte ergaben: für normale Kaninchen $45,4 \cdot 10^{-4} . \mathrm{Ohm}^{-1}$ und für normale Menschen $32,4 \cdot 10^{-4} . \mathrm{Ohm}^{-1}$.

Nicht unwichtig scheint mir die bei Kaninchen und Menschen zu erhebende Tatsache zu sein, dass eine Beeinflussung der Leitfähigkeit durch entzündliche Prozesse nicht konstatiert werden konnte. Wohl aber war eine Verletzung der Sklera von grösstem Einfluss auf die gemessenen Werte. Wie aus den Versuchen an den Kaninchenaugen, die mit und ohne Eröffnung der Sklera gemessen wurden, ersichtlich war, passierte der. Strom den Bulbus viel leichter bei direkter Einleitung in den Glaskörper als bei Auflegen der Elektroden auf die Sklera. Die viel geringere Konsistenz des Glaskörpers, die durch den grösseren Wassergehalt bedingte grössere Dissociationsmöglichkeit der Moleküle lassen das erklärlich erscheinen.

Ein an sich naheliegender Vergleich zwisehen der Glaskörperleitfähigkeit und der des Kammerwassers verbietet sich für meine Versuche, da die Messungen nicht unter gleichen Bedingungen vorgenommen wurden. Nicht uninteressant ist aber die Beobachtung, dass der durch die Entzündung veränderte Glaskörper ebenfalls eine gegen die Norm herabgesetzte Leitfähigkeit aufwies, ähnlich wie wir das beim Kammerwasser konstatieren konnten. Wir werden wohl nicht fehlgehen, wenn wir auch hier an den Einfluss des vermehrten Eiweissgehaltes in den erkrankten Augen denken.

Bei intakter Sklera war allerdings von einem Einfluss dieser herabgesetzten Leitfähigkeit des Glaskörpers auf die Grösse der Ge- 
samtleitfähigkeit des Bulbus nichts zu merken. Es wird nach alledem in allgemeinen die Leitfähigkeit des Bulbus bestimmt durch die Leitfähigkeit, welche die Sklera in dem durch die Form des Auges bedingten Lageverhältnis darbietet. Die Leitfähigkeit des Glaskörpers, anf die v. Ziemssen die von ihm als besonders gut erkannte Gesamtleitfähigkeit des Auges bezieht, spielt bei der Bestimmung des Gesamtwiderstandes in der Norm überhaupt keine Rolle; erst durch Veränderungen der Sklera, welche ihren Widerstand herabsetzen würden, oder durch Anwachsen des Widerstandes im Glaskörper bis etwa zur Grenze des Sklerawiderstandes würde die Leitfähigkeit des Glaskörpers Einfluss auf die Gesamtleitfähigkeit gewinnen.

Ist aber der Bulbus von den Lidern bedeckt, so verschwindet neben dem hohen Widerstand der Haut auch der der Sklera, der Widerstand der von dem Strome durchflossenen Partie ist jetzt lediglich bestimmt durch den Widerstand der den Elektroden anliegenden Hautteile.

Was nun endlich die Höhe der von mir gefundenen Leitfähigkeitswerte angeht, so möchte ich ausdrücklich darauf hinweisen, dass sie nur für die angewendete und genau geschilderte Versuchsanordnung gelten. „Denn am lebenden Organ können wir nicht die spezifische Leitfähigkeit bezogen auf den Centimeterwürfel der Substanz bestimmen und müssen daher darauf verzichten, Vergleichswerto losgelöst von den Versuchsbedingungen zu schaffen. Es ist also auch nicht möglich, ohne weiteres die von mir erhaltenen Werte mit denen von v. Ziemssen (1) zu vergleichen. Leider sind nun aber die Angaben bei v. Ziemssen über die Versuchsanordnung so ungenügend, dass wir auch nicht in der Lage sind, durch etwaige Korrekturen die Ungleichheiten in der Versuchsanordnung aufheben zu können. Es fehlen vor allem Angaben tiber den Querschnitt und den Widerstand der Elektroden und über die Stromstärke. Dass die andersartige Versuchsanordnung bei v. Ziemssen sicher eine grosse Rolle beim $Z$ ustandekommen seiner Werte gespielt haben muss, möchte ich schon aus der Grösse der Differenz zwischen seinen und meinen Zahlen schliessen. Rechnet man die von v. Ziemssen in SiemensEinheiten angegebenen Widerstandswerte in Ohm um, so erhält man: für den Widerstand des Bulbus: 2194,0 Ohm

" " " der Leber: 10904,0 "

Es wäre demnach die von ihm gefundene Leitfähigkeit des Bulbus etwa achtmal niedriger, als die sich aus meinen Messungen ergebende. 
144 E. Hertel, Untersuchungen über die elektrische Leitfähigkeit des Auges.

Beachtenswert ist, dass auch die v. Ziemssenschen Widerstandswerte für die Leber und Muskeln sowohl absolut als auch relativ genommen beträchtlich von denen bei andern Untersuchern abweichen. So fand z. B. Jellineck (4) für die Leber einen Widerstand von $900 \mathrm{Ohm}$, für Muskeln einen solchen von $1500 \mathrm{Ohm}$. Worin der Grund für v. Ziemssens hohe Werte gelegen ist, ist nicht mehr kontrollierbar; soviel aber ist zu sagen, dass nach meinen Untersuchungen der von ihm gefundene Widerstandswert für das Auge den Verhältnissen, wie sie in vivo vorliegen und in der Praxis event. yon Bedeutung sein können, weder nach der Versuchsanordnung noch nach dem Versuchsresultat entspricht.

\section{Literaturverzeichnis.}

1) v. Ziemssen, H., Die Elektrizität in der Medizin. Berlin 1887.

2) Erb, W., Handbuch der Elektrotherapie. Leipzig 1882.

3) Stintzing, R., Allgemeine Elektrotherapie der Erkrankungen des Nervensystems: im Handbuch der inneren Krankheiten von Penzoldt u. Stintzing. V. Bd. Jena 1903.

4) Jellineck, S., Elektropathologie. Stuttgart 1908.

5) Hamburger, J., Osmotischer Druck und Ionenlehre in den medizin. Wissenschaften. Wiesbaden 1902.

6) Eulenburg, A., Über episklerale Faradisation u. Galvanisation der Augenmuskeln. Zentralbl. f. prakt. Augenheilk. 1887. S. 67.

7) Lummer u. Kurlbaum, Wiedemanns Annalen. Bd. LX. S. 315.1897.

8) Kohlrausch, Holborn u. Diesselhorst, Neue Grundlagen für die Werte der Leitvermögen von Elektrolyten. Wiedemanns Annalen. Bd. LXIV. S. 417.1898.

9) Wessely, K., Zur Kenntnis der Wirkung lokaler Reize und lokaler Wärmeapplikation (mach Experimenten am Auge) Arch. f. klin. Chir. Bd. LXXI. S. 554.1903.

10) Bugarszky u. Tangl, Physikalisch-chemische Untersuchungen über die molekularen Konzentrationsverhältnisse des Blutserums. Pflügers Arch. $f$. d. ges. Physiol. Bd. LXXII. S. 531. 1898.

11) Wessely, K., Experimentelle Untersuchungen über Reizübertragung von einem Auge zum andern. v. Graefe's Arch. Bd. L, 1. S. 128.

12) Hammarsten, O., Über das Paraglobulin. Pflügers Arch.f. d. ges. Physiol. Bd. XVII. S. 459. 1878.

13) Römer, P., Die Pathogenese der Cataracta senilis vom Standpunkte der Serumforschung. Arch: f. Angenheilk. Bd. LVI. Erg.-Heft. 1907. 